This is an electronic reprint of the original article. This reprint may differ from the original in pagination and typographic detail.

Author(s): Taskinen, Sara; Miettinen, Jari; Nordhausen, Klaus

Title: A more efficient second order blind identification method for separation of uncorrelated stationary time series

Year: $\quad 2016$

Version:

Please cite the original version:

Taskinen, S., Miettinen, J., \& Nordhausen, K. (2016). A more efficient second order blind identification method for separation of uncorrelated stationary time series. Statistics and Probability Letters, 116, 21-26.

https://doi.org/10.1016/j.spl.2016.04.007

All material supplied via JYX is protected by copyright and other intellectual property rights, and duplication or sale of all or part of any of the repository collections is not permitted, except that material may be duplicated by you for your research use or educational purposes in electronic or print form. You must obtain permission for any other use. Electronic or print copies may not be offered, whether for sale or otherwise to anyone who is not an authorised user. 


\section{Accepted Manuscript}

A more efficient second order blind identification method for

separation of uncorrelated stationary time series

Sara Taskinen, Jari Miettinen, Klaus Nordhausen

PII:

S0167-7152(16)30025-6

DOI: $\quad$ http://dx.doi.org/10.1016/j.spl.2016.04.007

Reference: $\quad$ STAPRO 7588

To appear in: Statistics and Probability Letters

Received date: 18 February 2016

Revised date: 10 April 2016

Accepted date: 12 April 2016

Please cite this article as: Taskinen, S., Miettinen, J., Nordhausen, K., A more efficient second order blind identification method for separation of uncorrelated stationary time series. Statistics and Probability Letters (2016), http://dx.doi.org/10.1016/j.spl.2016.04.007

This is a PDF file of an unedited manuscript that has been accepted for publication. As a service to our customers we are providing this early version of the manuscript. The manuscript will undergo copyediting, typesetting, and review of the resulting proof before it is published in its final form. Please note that during the production process errors may be discovered which could affect the content, and all legal disclaimers that apply to the journal pertain. 


\title{
A more efficient second order blind identification method for separation of uncorrelated stationary time series
}

\author{
Sara Taskinen ${ }^{1 \mathrm{a}}$, Jari Miettinen ${ }^{\mathrm{a}}$, Klaus Nordhausen $^{\mathrm{b}, \mathrm{c}}$ \\ ${ }^{a}$ Department of Mathematics and Statistics, 40014 University of Jyväskylä, Finland \\ ${ }^{b}$ Department of Mathematics and Statistics, 20014 University of Turku, Finland \\ ${ }^{c}$ School of Health Sciences, 33014 University of Tampere, Finland
}

\begin{abstract}
The classical second order source separation methods use approximate joint diagonalization of autocovariance matrices with several lags to estimate the unmixing matrix. Based on recent asymptotic results, we propose a novel unmixing matrix estimator which selects the best lag set from a finite set of candidate sets specified by the user. The theory is illustrated by a simulation study.
\end{abstract}

Keywords: affine equivariane, asymptotic normality, joint diagonalization, linear process, minimum distance index, SOBI

\section{Introduction}

The second order source separation (SOS) model assumes that the observed $p$ time series are linear combinations of $p$ latent uncorrelated weakly (second-order) stationary time series with different time dependence structures. The aim is then to find an estimate for an unmixing matrix, which

\footnotetext{
${ }^{1}$ Corresponding author. Email: sara.taskinen@jyu.fi
} 
transforms the observed time series back to uncorrelated latent time series. The applications of second order source separation include, for example, the analysis of medical images and signals (EEG, MEG, fMRI) and financial time series (Hyvärinen et al., 2001; Comon and Jutten, 2010).

Classical unmixing matrix estimators for the SOS model use autocovariances and cross-autocovariances of the observed $p$ time series. The so-called algorithm for multiple unknown signals extraction (AMUSE) and secondorder blind identification (SOBI) algorithm for SOS models were suggested by Tong et al. (1990) and Belouchrani et al. (1997), respectively. In both methods the latent time series are found by performing joint diagonalization of autocovariance matrices. Later, in Miettinen et al. (2012, 2016a), corresponding unmixing matrix functionals and estimators were proposed and their statistical properties were derived. The limiting and finite sample performances of the estimators were investigated and it was noted that the performance of the estimators depends a lot on the selection of autocovariance matrices to be jointly diagonalized. See also Miettinen et al. (2014), Illner et al. (2015) and Miettinen (2015) for discussion on different algorithms for SOBI and the impact on the choice of autocovariances to be selected.

In this paper we address the problem of selecting the lags for the autocovariances to be diagonalized. We provide a two-stage estimation procedure which aims at finding the optimal set of autocovariance matrices for the SOBI estimate from the candidate sets specified by the user. The simulation studies show that our estimator, which is based on estimating asymptotic variances, is able to find the best set of autocovariance matrices when the length of the time series is sufficient. 
The paper is organized as follows. In Section 2 we recall the SOS model, classical functionals and estimators as well as theoretical results derived in Miettinen et al. (2016a). In Section 3, the procedure for a more efficient SOBI (eSOBI) is described, and in Section 4 its performance is compared with the classical SOBI estimators.

\section{Notations and definitions}

\subsection{Second order source separation (SOS) model}

Assume that the $p$-variate time series follows a Second Order Source Separation (SOS) model, that is, $\boldsymbol{x}=\left(\boldsymbol{x}_{t}\right)_{t=0, \pm 1, \pm 2, \ldots}$ is such that

$$
\boldsymbol{x}_{t}=\boldsymbol{\Omega} \boldsymbol{z}_{t}+\boldsymbol{\mu}, \quad t=0, \pm 1, \pm 2, \ldots
$$

where $\boldsymbol{\Omega}$ is a full-rank $p \times p$ mixing matrix, $\boldsymbol{\mu}$ is a $p$-variate location vector, which is considered as a nuisance parameter, and $\boldsymbol{z}=\left(\boldsymbol{z}_{t}\right)_{t=0, \pm 1, \pm 2, \ldots}$ is a $p$-variate latent time series satisfying

(A1) $E\left(\boldsymbol{z}_{t}\right)=0$ and $E\left(\boldsymbol{z}_{t} \boldsymbol{z}_{t}^{\prime}\right)=\boldsymbol{I}_{p}$.

(A2) $E\left(\boldsymbol{z}_{t} \boldsymbol{z}_{t+\tau}^{\prime}\right)=E\left(\boldsymbol{z}_{t+\tau} \boldsymbol{z}_{t}^{\prime}\right)=\boldsymbol{\Lambda}_{\tau}$ is diagonal for all $\tau=1,2, \ldots$

This is a semiparametric model as only moment assumptions on $\boldsymbol{z}$ are made. Assumption (A2) is needed for identifiability of $\boldsymbol{\Omega}$. Assumption (A1) fixes $\boldsymbol{\mu}$ and the scales of the components. After these assumptions, the signs and order of the components still remain unidentified. For practical purposes this is, however, sufficient. 


\subsection{SOS functionals based on autocovariance matrices}

The classical SOS functionals are based on the autocovariances and crossautocovariances of the $p$ time series. The most simple unmixing matrix functional is AMUSE (Algorithm for Multiple Unknown Signals) functional, which simultaneously diagonalizes the covariance matrix and an autocovariance matrix with selected lag $\tau$. The corresponding sample statistic, the so-called AMUSE estimator, was proposed in Tong et al. (1990).

The statistical properties of AMUSE estimators were studied recently in Miettinen et al. (2012). The drawback of the method is that it is highly sensitive to the selection of lag $\tau$. Motivated by this, Belouchrani et al. (1997) proposed the SOBI (Second Order Blind Identification) algorithm that aims at jointly approximately diagonalizing several autocovariance matrices.

Write now $\boldsymbol{S}_{\tau_{1}}, \ldots, \boldsymbol{S}_{\tau_{K}}$ for $K$ autocovariance matrices with distinct lags $\tau_{1}, \ldots, \tau_{K}$. In SOBI, the $p \times p$ unmixing matrix functional $\boldsymbol{\Gamma}=\left(\boldsymbol{\gamma}_{1}, \ldots, \boldsymbol{\gamma}_{p}\right)^{\prime}$ is then the matrix that maximizes

$$
\sum_{k=1}^{K}\left\|\operatorname{diag}\left(\boldsymbol{\Gamma} \boldsymbol{S}_{\tau_{k}} \boldsymbol{\Gamma}^{\prime}\right)\right\|^{2}=\sum_{j=1}^{p} \sum_{k=1}^{K}\left(\boldsymbol{\gamma}_{j}^{\prime} \boldsymbol{S}_{\tau_{k}} \boldsymbol{\gamma}_{j}\right)^{2}
$$

under the constraint $\boldsymbol{\Gamma} \boldsymbol{S}_{0} \boldsymbol{\Gamma}^{\prime}=\boldsymbol{I}_{p}$. Here we write $\operatorname{diag}(\boldsymbol{S})$ for a $p \times p$ diagonal matrix with the diagonal elements as in $\boldsymbol{S}$ and $\operatorname{off}(\boldsymbol{S})=\boldsymbol{S}-\operatorname{diag}(\boldsymbol{S})$.

The rows of $\boldsymbol{\Gamma}$ can be found either one by one (deflation-based approach) or simultaneously (symmetric approach). These two approaches were studied in Miettinen et al. (2014) and Miettinen et al. (2016a), respectively. In this paper we focus on symmetric SOBI as it is usually considered as the superior method. The corresponding estimating equations for the symmetric SOBI functional can be derived using the method of Lagrange multipliers. It was 
shown in Miettinen et al. (2016a) that the symmetric SOBI functional $\boldsymbol{\Gamma}$ can then be defined as follows.

Definition 1. The symmetric unmixing matrix functional $\boldsymbol{\Gamma}=\left(\boldsymbol{\gamma}_{1}, \ldots, \boldsymbol{\gamma}_{p}\right)^{\prime}$ solves the estimating equations

$$
\boldsymbol{\gamma}_{i}^{\prime} \boldsymbol{T}\left(\boldsymbol{\gamma}_{j}\right)=\boldsymbol{\gamma}_{j}^{\prime} \boldsymbol{T}\left(\boldsymbol{\gamma}_{i}\right) \quad \text { and } \quad \boldsymbol{\gamma}_{i}^{\prime} \boldsymbol{S}_{0} \boldsymbol{\gamma}_{j}=\delta_{i j}, \quad i, j=1, \ldots, p
$$

where $\boldsymbol{T}(\boldsymbol{\gamma})=\sum_{k=1}^{K}\left(\boldsymbol{\gamma}^{\prime} \boldsymbol{S}_{\tau_{k}} \boldsymbol{\gamma}\right) \boldsymbol{S}_{\tau_{k}} \boldsymbol{\gamma}$

The above estimating equations suggest a fixed-point algorithm for computing the SOBI estimator (Miettinen et al., 2016a). However, an equivalent solution can be computed faster using an algorithm for approximate joint diagonalization of matrices (Clarkson, 1988) which is based on Jacobi rotations. Algorithms for computing the AMUSE and SOBI estimates are provided in the R package JADE (Nordhausen et al., 2015; Miettinen et al., 2016b).

\subsection{Asymptotical properties of the SOS estimators}

The estimating equations allow us to study the asymptotical properties of the symmetric SOBI estimator defined as follows.

Definition 2. The unmixing matrix estimator $\hat{\boldsymbol{\Gamma}}=\left(\hat{\gamma}_{1}, \ldots, \hat{\gamma}_{p}\right)^{\prime}$, based on the sample autocovariance matrices $\hat{\boldsymbol{S}}_{0}, \hat{\boldsymbol{S}}_{\tau_{1}}, \ldots, \hat{\boldsymbol{S}}_{\tau_{K}}$, solves the estimating equations

$$
\hat{\boldsymbol{\gamma}}_{i}^{\prime} \hat{\boldsymbol{T}}\left(\hat{\boldsymbol{\gamma}}_{j}\right)=\hat{\boldsymbol{\gamma}}_{j}^{\prime} \hat{\boldsymbol{T}}\left(\hat{\boldsymbol{\gamma}}_{i}\right) \quad \text { and } \quad \hat{\boldsymbol{\gamma}}_{i}^{\prime} \hat{\boldsymbol{S}}_{0} \hat{\boldsymbol{\gamma}}_{j}=\delta_{i j}, \quad i, j=1, \ldots, p
$$

where $\hat{\boldsymbol{T}}(\boldsymbol{\gamma})=\sum_{k=1}^{K}\left(\boldsymbol{\gamma}^{\prime} \hat{\boldsymbol{S}}_{\tau_{k}} \boldsymbol{\gamma}\right) \hat{\boldsymbol{S}}_{\tau_{k}} \boldsymbol{\gamma}$

Now assume that the observed times series follows the SOS model (1) and assume (wlog) that $\boldsymbol{\mu}=\mathbf{0}$. We also assume that 
(A3) the diagonal elements of $\sum_{k=1}^{K} \Lambda_{\tau_{k}}^{2}$ are strictly decreasing.

(A4) $\boldsymbol{\Omega}=\boldsymbol{I}_{p}$ and $\sqrt{T}\left(\hat{\boldsymbol{S}}_{\tau_{k}}-\boldsymbol{\Lambda}_{\tau_{k}}\right)=O_{p}(1), k=0,1, \ldots, K$ as $T \rightarrow \infty$.

Assumption (A3) guarantees the identifiability of the mixing matrix and fixes the order of the component time series in the SOS model. Assumption (A4) is needed for consistency. Miettinen et al. (2016a) then proved the following result.

Theorem 1. Under (A1)-(A4), $\hat{\boldsymbol{\Gamma}}=\left(\hat{\gamma}_{1}, \ldots, \hat{\gamma}_{p}\right)^{\prime} \rightarrow_{p} \boldsymbol{I}_{p}$. Further, if the joint limiting distribution of

$$
\sqrt{T}\left[\operatorname{vec}\left(\hat{\boldsymbol{S}}_{0}, \hat{\boldsymbol{S}}_{\tau_{1}}, \ldots, \hat{\boldsymbol{S}}_{\tau_{K}}\right)-\operatorname{vec}\left(\boldsymbol{I}_{p}, \boldsymbol{\Lambda}_{\tau_{1}}, \ldots, \boldsymbol{\Lambda}_{\tau_{K}}\right)\right]
$$

is a singular $(K+1) p^{2}$-variate normal distribution with mean value zero, then the joint limiting distribution of $\sqrt{T} \operatorname{vec}(\hat{\boldsymbol{\Gamma}}-\boldsymbol{\Gamma})$ is a singular $p^{2}$-variate normal distribution.

The SOBI estimator is affine equivariant which means that $\hat{\Gamma} \boldsymbol{\Omega}$ and the estimated latent time series do not depend on the mixing matrix $\Omega$. This implies that neither the limiting distribution of $\sqrt{T} \operatorname{vec}\left(\hat{\boldsymbol{\Gamma}} \boldsymbol{\Omega}-\boldsymbol{I}_{p}\right)$ depends on $\boldsymbol{\Omega}$, and also the limiting distribution of $\sqrt{T} \operatorname{vec}(\hat{\boldsymbol{\Gamma}}-\boldsymbol{\Gamma})$ is obtained easily for arbitrary $\Omega$.

The limiting joint normality of sample autocovariance matrices naturally depends on the distribution of the latent $p$-variate time series. In Miettinen et al. (2012), the joint limiting normality was proven assuming that $\boldsymbol{z}_{t}$ are uncorrelated multivariate linear processes, that is,

$$
\boldsymbol{z}_{t}=\sum_{j=-\infty}^{\infty} \boldsymbol{\Psi}_{j} \boldsymbol{\epsilon}_{t-j}
$$


where $\boldsymbol{\epsilon}_{t}$ are standardized iid $p$-vectors (with exchangeable and marginally symmetric components with finite fourth moments) and $\boldsymbol{\Psi}_{j}, j=0, \pm 1, \pm 2, \ldots$, are diagonal matrices satisfying $\sum_{j=-\infty}^{\infty} \Psi_{j}^{2}=\boldsymbol{I}_{p}$. Notice that for example all causal ARMA processes can be written as linear processes. For the limiting covariance matrices of $\sqrt{T} \operatorname{vec}(\hat{\boldsymbol{\Gamma}}-\boldsymbol{\Gamma})$, see then Miettinen et al. (2016a).

In the case of linear processes, the limiting variances of $\sqrt{T} \operatorname{vec}\left(\hat{\boldsymbol{\Gamma}} \boldsymbol{\Omega}-\boldsymbol{I}_{p}\right)$ can be estimated from the data, even if $\Omega$ is unknown. Further, when $\boldsymbol{\epsilon}_{t}$ are Gaussian, the limiting variances depend on $\boldsymbol{\Psi}_{j}, j=0, \pm 1, \pm 2, \ldots$, only through the autocovariance matrices of the process. Hence, the estimation of the limiting variances is relatively simple in the Gaussian case, the only dilemma being the infinite number of autocovariance matrices of $\boldsymbol{z}$ in the formulas. The number of autocovariances, $M$, to be included in the estimation procedure is upper bounded by $T-1$, where $T$ is the sample size, and lower bounded by the largest lag $\tau_{K}$. The target $M$ is the largest lag for whch the autocovariance matrix of $\boldsymbol{z}$ is nonzero. If $\boldsymbol{\epsilon}_{t}$ are not Gaussian, the formulas for the limiting variances include $\boldsymbol{\Psi}_{j}, j=0, \pm 1, \pm 2, \ldots$, as well as the fourth moments of $\boldsymbol{\epsilon}_{t}$. The estimation thus becomes more cumbersome. Using the R package BSSasymp (Miettinen et al., 2015), the covariance matrix of $\sqrt{T} \operatorname{vec}(\hat{\boldsymbol{\Gamma}}-\boldsymbol{\Gamma})$ can be estimated both under the Gaussianity assumption and without it.

\section{A more efficient SOBI estimator}

The choice of autocovariance matrices to be jointly diagonalized in SOBI is known to affect the efficiencies of SOBI estimators (Tang et al., 2005; Miettinen et al., 2012, 2016a). However, only few ad-hoc guidelines for the 
autocovariance matrix selection are provided in the literature (Tang et al., 2005 , e.g.). Motivated by this we propose the eSOBI estimator, which makes use of the asymptotical results described in the previous section.

In Ilmonen et al. (2010) it was shown that the sum of the limiting variances of the off-diagonal elements of $\sqrt{T} \operatorname{vec}\left(\hat{\boldsymbol{\Gamma}} \boldsymbol{\Omega}-\boldsymbol{I}_{p}\right)$ provide a global measure of variation of $\hat{\boldsymbol{\Gamma}}$. We thus propose an estimation procedure in which we go through sets of autocovariance matrices specified by the user, and choose the set which yields the most efficient unmixing matrix estimator, that is, the one with smallest estimated global measure of variation. Let $\boldsymbol{X}=\left(\boldsymbol{x}_{1}, \ldots, \boldsymbol{x}_{T}\right)$ denote the (centered) data matrix. The estimator is found via the following simple algorithm:

1. Choose $L$ lag sets $\left\{\mathrm{T}_{1}, \mathrm{~T}_{2}, \ldots, \mathrm{T}_{L}\right\}$.

2. For each lag set $\mathrm{T}_{l}, l=1, \ldots, L$, compute $\hat{\boldsymbol{\Gamma}}_{l}$ and $\hat{\boldsymbol{Z}}^{l}=\hat{\boldsymbol{\Gamma}}_{l} \boldsymbol{X}$, and estimate the asymptotic covariance matrix of $\sqrt{T} \operatorname{vec}\left(\hat{\boldsymbol{\Gamma}}_{l} \boldsymbol{\Omega}-\boldsymbol{I}_{p}\right)$ based on $\hat{Z}^{l}$.

3. The efficient SOBI estimator is the one that minimizes the sum of the limiting variances of the off-diagonal elements of $\sqrt{T} \operatorname{vec}\left(\hat{\boldsymbol{\Gamma}} \boldsymbol{\Omega}-\boldsymbol{I}_{p}\right)$.

The affine equivariance of the estimator follows from that of the regular SOBI, since the choice of the lag set is only based on the estimated latent time series $\hat{Z}^{l}$ which do not depend on the mixing matrix. We also consider a procedure where only one $\hat{\boldsymbol{\Gamma}}_{l}$ and one $\hat{\boldsymbol{Z}}^{l}$ is computed in step 2, and the asymptotic covariance matrices for all lag sets are estimated using $\hat{\boldsymbol{Z}}^{l}$. This approach is obviously faster to compute. In Section 4 we compare the efficiency of the two variants. 
As instead of unbiased estimation of the limiting variances, the main interest is in finding the smallest sum of the limiting variances, we recommend that the assumption on the source components being Gaussian processes is made. This results in substantial reductions in computation times and is justified by the fact that the effect of non-Gaussianity to the limiting variances is rather small and almost similar for each lag set (Miettinen et al., $2012,2014)$.

In addition to the selection of the lag set, one could use the asymptotical results to choose between the symmetric and deflation-based SOBI or to choose the diagonality criterion (Miettinen, 2015). However, when combined with the selection of the lags, we regard the gain which can be attained from these choices too small as compared to the extra computational burden. Therefore we focus here only on the standard symmetric SOBI estimator. The R package BSSasymp (Miettinen et al., 2015) provides a function for the computation of the eSOBI estimate, and also tools to construct more complicated versions.

\section{Simulation studies}

In this section we compare the performance of the eSOBI estimator to that of classical SOBI estimator using simulation studies. As a performance index we use the minimum distance index (MDI, Ilmonen et al., 2010), which compares the estimated mixing matrix to the true one. The minimum distance index is defined as

$$
\hat{D}=D(\hat{\boldsymbol{\Gamma}} \boldsymbol{\Omega})=\frac{1}{\sqrt{p-1}} \inf _{\boldsymbol{C} \in \mathcal{C}}\left\|\boldsymbol{C} \hat{\boldsymbol{\Gamma}} \boldsymbol{\Omega}-\boldsymbol{I}_{p}\right\|
$$


where $\|\cdot\|$ is the matrix (Frobenius) norm and $\mathcal{C}$ is the set of $p \times p$ matrices which have exactly one non-zero element on each row and column. The index is invariant with respect to the change of the mixing matrix, and it is scaled so that $0 \leq \hat{D} \leq 1$. The smaller the MDI-value, the better is the performance.

To compare the performances, multivariate time series were generated in R 3.2.3 (R Core Team, 2015) from the following four models with different choices of $T$ and with 2000 repetitions. In all simulation settings, the mixing matrix was $\Omega=\boldsymbol{I}_{p}$ as due to affine equivariance, this has no effect on the results.

(A) $A R(1), A R(2), A R(3)$ and $A R M A(1,1)$ time series with Gaussian innovations.

(B) $A R(1), A R(2), M A(15)$ and $M A(15)$ time series with exponentially, $t_{5}$, normally and uniformly distributed innovations, respectively.

(C) The same coefficients as in $(\mathrm{A})$ with multivariate $t_{5}$-distributed innovations.

(D) Time series of models (A) and (B) combined.

Notice that in models (A), (B) and (D) the components of the time series are mutually independent and in model $(\mathrm{C})$ they are only uncorrelated, still satisfying the assumption (A2). The eSOBI estimators were computed using the following 17 candidate sets of lags:

Hence the lag sets have different lengths and consider different lag combinations. Set $\mathrm{T}_{1}$ yields the AMUSE type of estimate, and set $\mathrm{T}_{11}$ is the 


$$
\begin{array}{ll}
\mathrm{T}_{1}=\{1\} & \mathrm{T}_{13}=\{1,2, \ldots, 50\} \\
\mathrm{T}_{2}=\{1,2\} & \mathrm{T}_{14}=\{1,2, \ldots, 10,12, \ldots, 20\} \\
\vdots & \mathrm{T}_{15}=\{5,6, \ldots, 10,12, \ldots, 20,25, \ldots, 50\} \\
\mathrm{T}_{10}=\{1,2, \ldots, 10\} & \mathrm{T}_{16}=\{2,4, \ldots, 20\} \\
\mathrm{T}_{11}=\{1,2, \ldots, 12\} & \mathrm{T}_{17}=\{1,2,3,5,7,11,13,17,19\} \\
\mathrm{T}_{12}=\{1,2, \ldots, 20\} &
\end{array}
$$

default set in many papers considering SOBI estimator. Tang et al. (2005) argue that in the context of EEG data, also large lags are important. This motivates us for using the sets $T_{13}$ and $T_{15}$ as candidate sets. Notice that our $\mathrm{R}$ function eSOBI in the package BSSasymp allows the user to include all those candidate lag sets into the collection he/she considers useful for the application at hand.

Four eSOBI estimators are computed in the simulation studies. The candidate sets are fixed, but the estimators differ in how the asymptotic variances are estimated. The first estimator is computed as described in the algorithm in Section 3, that is, the initial SOBI estimate is computed separately for each lag set. For the other eSOBI estimators, the asymptotic variances for all lag sets are estimated using a common initial SOBI estimate. The initial SOBI estimates use sets $\mathrm{T}_{1}, \mathrm{~T}_{11}$ and $\mathrm{T}_{15}$, respectively. The eSOBI estimators are compared to AMUSE with $\tau=1$ and SOBI with $\mathrm{T}_{2}, \mathrm{~T}_{5}, \mathrm{~T}_{11}$ and $\mathrm{T}_{13}$. Also the minimum of $T(p-1) \hat{D}^{2}$ over all candidate sets is computed. Notice that in all our computations the maximum number of autocovariances, $\mathrm{M}$, to compute the asymptotic quantities was set to 200 .

The results in Figure 1 show that the choice of the common initial es- 
timate has an evident effect in models (A), (C) and (D) when $T$ is small, but all eSOBI estimates converge to the same values as $T$ increases. It is also worth mentioning that when $T$ is sufficiently large, then always the set, which was theoretically the best out of the candidate sets, was picked for each eSOBI. This means in setting $(A) T_{2}$, setting (B) $\mathrm{T}_{12}$, setting $(\mathrm{C}) \mathrm{T}_{2}$ and setting (D) $\mathrm{T}_{2}$. Notice that the theoretically best set is not the best in each repetition and therefore the average of the minimum of $T(p-1) \hat{D}^{2}$ over all candidate sets is below that of the eSOBI estimator.

\section{Conclusions}

SOBI is a popular blind source separation method, and widely used for example in the analysis of biomedical signals. The selection of lags for the autocovariance matrices to be jointly diagonalized in SOBI has a significant effect on the performance of the method. However, only ad hoc guidelines for lag selection are given in the literature. In this paper we propose a more efficient version of the (symmetric) SOBI method. The more efficient SOBI method (eSOBI) goes through all the lag sets specified by the user, and chooses the best one using asymptotic considerations. In our simulation studies we showed the excellent performance of eSOBI. Hence we advice users to use subject knowledge to select a rich set of candidate sets of lags. The eSOBI will then select the best one. If a common initial estimate is used and the gaussianity assumption is made when estimating the asymptotic variances, the computational costs are quite small as compared to the gained efficiency. 


\section{Acknowledgements}

The authors wish to thank the referee for valuable comments. This work was supported by the Academy of Finland (grants 251965, 256291 and 268703).

\section{References}

Belouchrani, A., Abed-Meraim, K., Cardoso, J.-F. and Moulines, E., 1997. A blind source separation technique using second-order statistics. IEEE Trans. Signal Proc. 45, 434-444.

Clarkson, D.B., 1988. A least squares version of algorithm AS 211: The F-G diagonalization algorithm. Applied Statistics 37, 317-321.

Comon P. and Jutten C., 2010. Handbook of Blind Source Separation. Independent Component Analysis and Applications. Academic Press, Amsterdam.

Hyvärinen, A. Karhunen, J. and Oja, E., 2001. Independent Component Analysis. John Wiley and Sons, New York.

Illner, K., Miettinen, J., Fuchs,C., Taskinen, S., Nordhausen, K., Oja, H. and Theis, F.J., 2015. Model selection using limiting distributions of secondorder blind source separation algorithms. Signal Proecess. 113, 95-103.

Ilmonen, P., Nordhausen, K., Oja, H. and Ollila, E., 2010. A new performance index for ICA: properties computation and asymptotic analysis, in Vigneron, V., Zarzoso, V., Moreau, E., Gribonval, R. and Vincent, E. 
(Eds.), Latent Variable Analysis and Signal Separation. Springer, Heidelberg, 229-236

Miettinen, J., 2015. Alternative diagonality criteria for SOBI, in Nordhausen, K. and Taskinen, S. (Eds), Modern Nonparametric, Robust and Multivariate Methods. Festschrift in Honour of Hannu Oja, Springer, Cham, $455-469$.

Miettinen, J., Nordhausen, K., Oja, H. and Taskinen, S., 2012. Statistical properties of a blind source separation estimator for stationary time series. Statist. Prob. Lett. 82, 1865-1873.

Miettinen, J., Nordhausen, K., Oja, H. and Taskinen, S., 2014. Deflationbased separation of uncorrelated stationary time series. J. Multiv. Anal. $123,214-227$.

Miettinen, J., Illner, K., Nordhausen, K., Oja, H., Taskinen, S. and Theis, F., 2016a. Separation of uncorrelated stationary time series using autocovariance matrices. J. Time Ser. Anal., 37, 337-354.

Miettinen, J., Nordhausen, K., Oja, H. and Taskinen, S., 2015. BSSasymp: Asymptotic Covariance Matrices of Some BSS Mixing and Unmixing Matrix Estimates. $\mathrm{R}$ package version 1.1-1. http://cran.rproject.org/web/packages/BSSasymp.

Miettinen, J., Nordhausen, K. and Taskinen, S., 2016b. Blind Source Separation Based on Joint Diagonalization in R: The Packages JADE and BSSasymp. Conditionally accepted for publication in the Journal of Statistical Software. 
Nordhausen, K., Cardoso, J.-F., Miettinen, J., Oja, H., Ollila, E. and Taskinen, S., 2015. JADE: Blind Source Separation Methods Based on Joint Diagonalization and Some BSS Performance Criteria. R package version 1.9-93. http://cran.r-project.org/web/packages/JADE.

R Core Team, 2015. R: A Language and Environment for Statistical Computing. R Foundation for Statistical Computing, Vienna, Austria. URL https://www.R-project.org/.

Tang, A.C., Liu, J.-Y. and Sutherland M.T., 2005. Recovery of correlated neuronal sources from EEG: The good and bad ways of using SOBI. NeuroImage $7,507-519$.

Tong, L., Soon, V.C., Huang, Y.F. and Liu, R., 1990. AMUSE: a new blind identification algorithm, in Proceedings of IEEE International Symposium on Circuits and Systems 1990, pp. 1784-1787. 

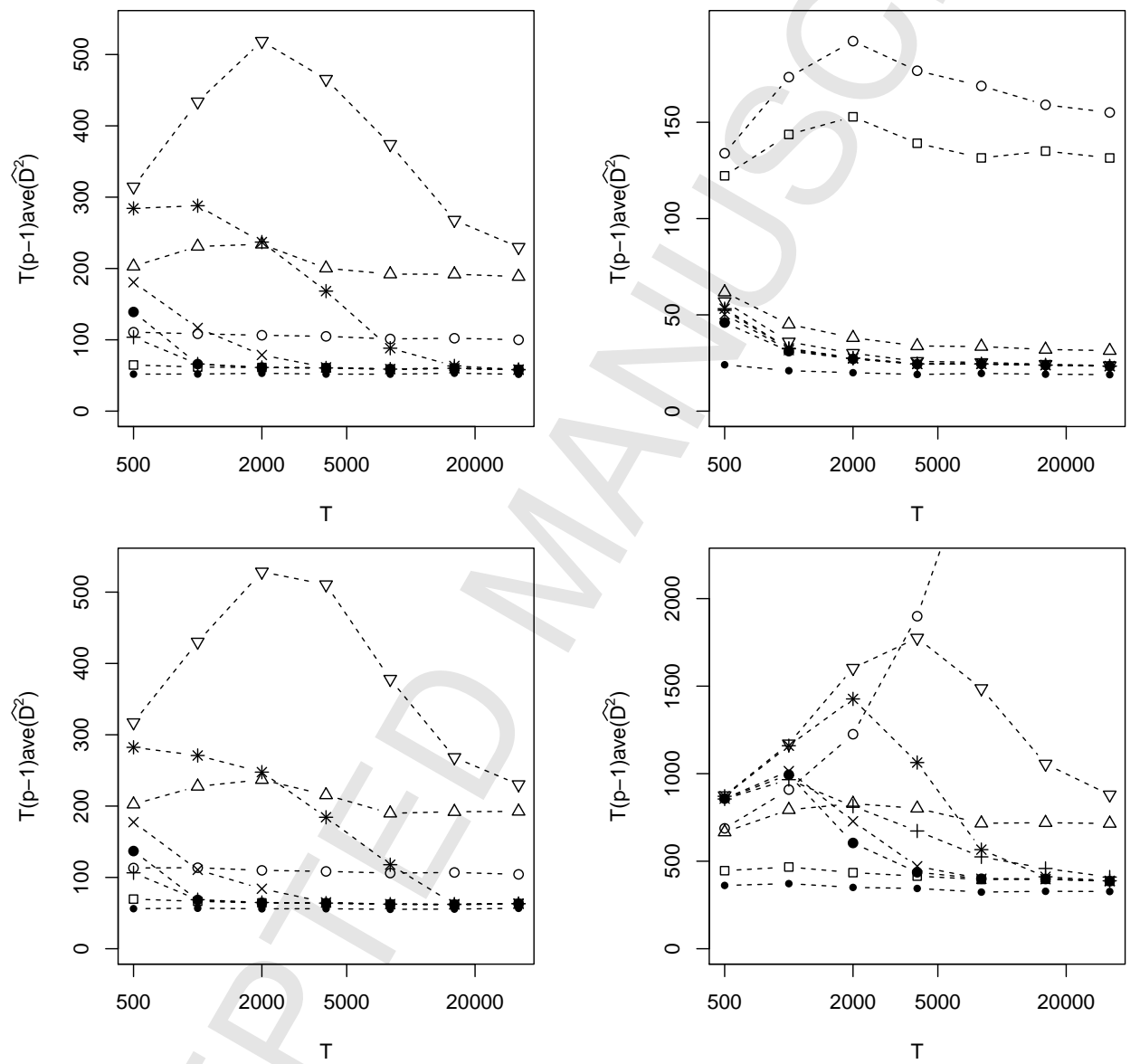

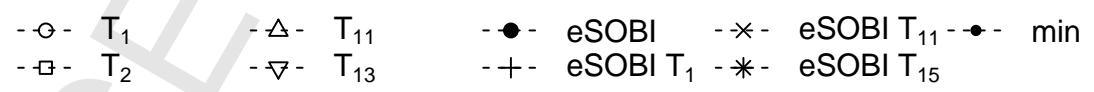

Figure 1: The averages of $T(p-1) \hat{D}^{2}$ for different estimators. 